\title{
Correction to: Healthcare professionals' perspectives on working conditions, leadership, and safety climate: a cross- sectional study
}

\author{
Anke Wagner ${ }^{1 *}$, Monika A. Rieger ${ }^{1}$, Tanja Manser ${ }^{2}$, Heidrun Sturm¹, Juliane Hardt ${ }^{3,4,5}$, Peter Martus ${ }^{3}$, \\ Constanze Lessing ${ }^{7}$, Antje Hammer ${ }^{6}$ and on behalf of the WorkSafeMed Consortium
}

\section{Correction to: BMC Health Serv Res \\ https://doi.org/10.1186/s12913-018-3862-7}

In the original publication of this article [1], the authors missed that reverse coding was necessary for the item "Do you work separate from your colleagues?" before calculating the scale 'social relations'. So they corrected the analysis accordingly. The results with the revised scale show that there are no longer any significant differences between nurses and physicians with regard to this scale.

This error (scale social relations) affects the following parts of our manuscript:

'Methods' section:

Old version: We also adapted one scale from the Copenhagen Burnout Inventory (client-related burnout) to measure patient-related burnout [54]. Before calculating scale scores for each dimension and in ac- cordance with the recommended COPSOQ transformation [52], scales were transformed into scores ranging from 0 (minimum value, "do not agree at all") to 100 points (maximum value, "fully agree").

Correction: We also adapted one scale from the Copenhagen Burnout Inventory (client-related burnout) to measure patient-related burnout [54]. Before scale calculation, reverse coding was carried out for one item ("Do you work separate from your colleagues?"). Scale calculation was done in accordance with the recommended COPSOQ transformation [52], scales were transformed into scores ranging from 0 (minimum value, "do not agree at all") to 100 points (maximum value, "fully agree").

The original article can be found online at https://doi.org/10.1186/s12913018-3862-7

* Correspondence: anke.wagner@med.uni-tuebingen.de

'Institute of Occupational and Social Medicine and Health Services Research, University Hospital of Tübingen, Wilhelmstraße 27, 72074 Tübingen, Germany Full list of author information is available at the end of the article

(c) The Author(s). 2020 Open Access This article is distributed under the terms of the Creative Commons Attribution 4.0 International License (http://creativecommons.org/licenses/by/4.0/), which permits unrestricted use, distribution, and reproduction in any medium, provided you give appropriate credit to the original author(s) and the source, provide a link to the Creative Commons license, and indicate if changes were made. The Creative Commons Public Domain Dedication waiver (http://creativecommons.org/publicdomain/zero/1.0/) applies to the data made available in this article, unless otherwise stated. 


\section{'Result' section:}

Old version: There were no statistically significant differences between the two professional groups in the four scales predictability, role clarity, feedback, and sense of community.

Correction: There were no statistically significant differences between the two professional groups in the five scales predictability, role clarity, feedback, social relations, and sense of community.

Old version: We identified significant differences with small or medium effects in three scales (social support: $\mathrm{d}=-.15$, role conflicts: $\mathrm{d}=-.31$, and social relations: $\mathrm{d}=.40)$.

Correction: We identified significant differences with small or medium effects in two scales (social support: $\mathrm{d}=-.15$, and role conflicts: $\mathrm{d}=-.31$ ).

Old version: Physicians rated items on the scale social relations more positively $(51.5 \pm 15.1)$ than the nurses $(45.0 \pm 17.0)$.

Correction: Values for the scale social relations were relatively high for both physicians $(54.8 \pm 20.7)$ and nurses $(55.5 \pm 20.2)$. There was no significant difference between the two professional groups in rating the scale social relations.

\section{'Discussion' section:}

Old version: Our study found significant differences between the two professional groups in 12 out of 17 scales. Nine scales (influence at work, degree of freedom at work, possibilities for development, meaning of work, workplace commitment, role conflicts, social relations, job satisfaction, and the additional scale patient-related burnout) were significantly more positively assessed by physicians than the nursing staff),

Corrected version: Our study found significant differences between the two professional groups in 11 out of 17 scales. Eight scales (influence at work, degree of freedom at work, possibilities for development, meaning of work, workplace commitment, role conflicts, job satisfaction, and the additional scale patient-related burnout) were significantly more positively assessed by physicians than the nursing staff.

Revised Table 3

We corrected the values for the scale "Social relations". We also detected some minor errors with no consequences and corrected them too (for the following scales or single items: "Emotional demands", "Teamwork within units", "My direct supervisor focuses more on patient safety than a year ago", "Hospital management openly addresses problems concerning patient safety in our hospital", "Hospital management focuses more on patient safety than a year ago" and "My direct supervisor openly addresses problems concerning occupational safety in our hospital".

Table 3 with the corrected values is shown below:

Table 3 Descriptive statistics, results of the student's t test and effect size comparing answers by nurses and physicians

\begin{tabular}{|c|c|c|c|c|c|}
\hline Psychosocial working conditions & $\begin{array}{l}\text { Interpretation } \\
(0=\text { minimum value, } \\
100=\text { maximum value })\end{array}$ & $\begin{array}{l}\text { Mean (SD) } \\
\text { (nurses=564) }\end{array}$ & $\begin{array}{l}\text { Mean (SD) } \\
\text { (physicians=380) }\end{array}$ & (df) t-value ${ }^{1}$ & $d_{\text {Cohen }}$ \\
\hline \multicolumn{6}{|c|}{ Copenhagen Psychosocial Questionnaire (COPSOQ) } \\
\hline Quantitative demands & high=negative & $66.5(13.5)$ & $71.9(13.9)$ & (942) $-5.974^{*}$ & 0.40 \\
\hline Emotional demands & high=negative & $64.4(18.3)$ & $64.6(16.5)$ & (866) -0.206 & 0.01 \\
\hline Work-privacy-conflict & high=negative & $61.3(24.4)$ & $68.7(25.1)$ & $(942)-4.497^{*}$ & 0.30 \\
\hline Influence at work & high=positive & $36.3(17.3)$ & $38.8(20.8)$ & (710) $-2.006^{*}$ & 0.13 \\
\hline Degree of freedom at work & high=positive & $36.0(15.9)$ & $46.2(20.0)$ & $(687)-8.373^{*}$ & 0.58 \\
\hline Possibilities for development & high=positive & $71.6(15.7)$ & $79.6(14.2)$ & $(942)-8.032^{*}$ & 0.53 \\
\hline Meaning of work & high=positive & $77.7(16.6)$ & $82.9(16.1)$ & $(942)-4.753^{*}$ & 0.32 \\
\hline Workplace commitment & high=positive & $48.4(18.8)$ & $61.3(19.2)$ & $(942)-10.220^{*}$ & 0.68 \\
\hline Predictability & high=positive & $53.3(16.4)$ & $52.5(19.3)$ & (720) 0.710 & -0.05 \\
\hline Role clarity & high=positive & $73.5(14.5)$ & $72.5(16.5)$ & (740) 1.027 & -0.07 \\
\hline Role conflicts & high=negative & $50.6(17.2)$ & $45.1(18.4)$ & (942) $4.611^{*}$ & -0.31 \\
\hline Feedback & high=positive & $41.9(21.0)$ & $41.0(21.5)$ & (942) 0.632 & -0.04 \\
\hline Social support & high=positive & $66.7(17.0)$ & $64.2(17.0)$ & (942) $2.169^{*}$ & -0.15 \\
\hline Social relations & high=positive & $55.5(20.2)$ & $54.8(20.7)$ & (942) 0.512 & -0.03 \\
\hline Sense of community & high=positive & $77.8(15.2)$ & $76.7(15.1)$ & (942) 1.096 & -0.07 \\
\hline \multicolumn{6}{|c|}{ Outcome scale - Copenhagen Psychosocial Questionnaire (COPSOQ) } \\
\hline Job satisfaction & high=positive & $67.5(10.2)$ & $73.4(12.0)$ & $(942)-8.135^{*}$ & 0.54 \\
\hline
\end{tabular}


Table 3 Descriptive statistics, results of the student's t test and effect size comparing answers by nurses and physicians (Continued)

\begin{tabular}{|c|c|c|c|c|c|}
\hline \multicolumn{6}{|c|}{ Outcome scale - Copenhagen Burnout Inventory (CBI, adapted client-related burnout) } \\
\hline Patient related burnout & high=negative & $36.5(17.6)$ & $28.0(16.5)$ & (942) $7.464^{*}$ & -0.50 \\
\hline Leadership & $\begin{array}{l}\text { Interpretation } \\
(0 / 1=\text { minimum value, } \\
100 / 5=\text { =maximum value) }\end{array}$ & $\begin{array}{l}\text { Mean }(S D) \\
\text { (nurses=543) }\end{array}$ & $\begin{array}{l}\text { Mean (SD) } \\
\text { (physicians=369) }\end{array}$ & (df) t-value ${ }^{1}$ & $d_{\text {Cohen }}$ \\
\hline \multicolumn{6}{|l|}{ Transformational Leadership Inventory (TLI short) } \\
\hline Transformational leadership & $5=$ positive & $3.1(0.8)$ & $3.2(0.8)$ & (910) -1.605 & 0.13 \\
\hline \multicolumn{6}{|l|}{ Copenhagen Psychosocial Questionnaire (COPSOQ) } \\
\hline Quality of leadership & high=positive & $53.8(22.7)$ & $49.2(22.9)$ & (910) $3.031^{*}$ & -0.20 \\
\hline Patient safety climate & $\begin{array}{l}\text { Interpretation } \\
(1=\text { minimum value, } \\
5=\text { maximum value })\end{array}$ & $\begin{array}{l}\text { Mean }(S D) \\
\text { (nurses=558) }\end{array}$ & $\begin{array}{l}\text { Mean (SD) } \\
\text { (physicians=373) }\end{array}$ & (df) t-value $e^{1}$ & $d_{\text {Cohen }}$ \\
\hline \multicolumn{6}{|l|}{ Hospital Survey on Patient Safety Culture (HSPSC-D) } \\
\hline Staffing & $5=$ positive & $2.4(0.8)$ & $2.8(0.8)$ & (929) $-7.721^{*}$ & 0.50 \\
\hline Organizational learning & $5=$ positive & $3.0(0.7)$ & $3.1(0.7)$ & (762) -1.366 & 0.14 \\
\hline Communication openness & $5=$ positive & $3.7(0.6)$ & $3.4(0.7)$ & (758) $6.010^{*}$ & -0.47 \\
\hline Feedback \& communication about error & $5=$ positive & $3.4(0.8)$ & $3.3(0.9)$ & (929) 1.519 & -0.12 \\
\hline Nonpunitive response to error & $5=$ positive & $3.3(0.8)$ & $3.5(0.8)$ & (929) $-3.746^{*}$ & 0.25 \\
\hline Teamwork within units & $5=$ positive & $3.3(0.6)$ & $3.4(0.6)$ & (929) -1.326 & 0.17 \\
\hline Teamwork across units & $5=$ positive & $3.0(0.6)$ & $3.1(0.7)$ & (698) $-3.316^{*}$ & 0.16 \\
\hline Handoffs \& transitions & $5=$ positive & $3.2(0.6)$ & $2.9(0.7)$ & (713) $5.702^{*}$ & -0.47 \\
\hline Supervisor/ manager expectations & $5=$ positive & $3.4(0.7)$ & $3.3(0.7)$ & (929) 1.020 & -0.14 \\
\hline Management support for patient safety & $5=$ positive & $2.6(0.8)$ & $3.0(0.8)$ & (929) $-5.797^{*}$ & 0.50 \\
\hline \multicolumn{6}{|c|}{ Outcome scales - Hospital Survey on Patient Safety Culture (HSPSC-D) } \\
\hline Frequency of event reported & $5=$ positive & $3.0(1.1)$ & $2.9(0.9)$ & (874) 1.053 & -0.10 \\
\hline Overall perceptions of patient safety & $5=$ positive & $2.9(0.7)$ & $3.3(0.8)$ & (929) $-7.782^{*}$ & 0.54 \\
\hline Patient safety grade & $1=$ positive & $2.9(0.8)$ & $2.6(0.7)$ & (929) $7.456^{*}$ & -0.39 \\
\hline Safety grade in the medication process & $1=$ positive & $3.0(0.8)$ & $2.8(0.7)$ & (831) $5.065^{*}$ & -0.26 \\
\hline Patient safety climate & $\begin{array}{l}\text { Interpretation } \\
(1=\text { minimum value, } \\
5=\text { maximum value })\end{array}$ & $\begin{array}{l}\text { Mean }(S D) \\
\text { (nurses=543) }\end{array}$ & $\begin{array}{l}\text { Mean (SD) } \\
\text { (physicians=369) }\end{array}$ & (df) t-value ${ }^{1}$ & $d_{\text {Cohen }}$ \\
\hline \multicolumn{6}{|l|}{ TWINS Patient Safety } \\
\hline Supervisor support for patient safety & $5=$ positive & $3.4(0.8)$ & $3.5(0.7)$ & (910) $-1.996^{*}$ & 0.13 \\
\hline $\begin{array}{l}\text { My direct supervisor openly addresses problems } \\
\text { concerning patient safety in our hospital }\end{array}$ & $5=$ positive & $3.3(0.9)$ & $3.3(1.0)$ & (729) -0.865 & 0.00 \\
\hline $\begin{array}{l}\text { My direct supervisor focuses more on patient } \\
\text { safety than a year ago }\end{array}$ & $5=$ positive & $2.8(0.9)$ & $2.8(1.0)$ & (735) -0.027 & 0.00 \\
\hline $\begin{array}{l}\text { It is important to my direct supervisor that our } \\
\text { hospital pays great attention to patient safety }\end{array}$ & $5=$ positive & $3.4(0.9)$ & $3.5(0.9)$ & (910) -1.509 & 0.11 \\
\hline $\begin{array}{l}\text { Hospital management openly addresses problems } \\
\text { concerning patient safety in our hospital }\end{array}$ & $5=$ positive & $2.7(0.8)$ & $3.0(0.9)$ & (910) $-4.188^{*}$ & 0.36 \\
\hline $\begin{array}{l}\text { Hospital management focuses more on patient } \\
\text { safety than a year ago }\end{array}$ & $5=$ positive & $2.7(0.9)$ & $2.8(0.9)$ & (910) $-2.758^{*}$ & 0.11 \\
\hline $\begin{array}{l}\text { It is important to the Hospital management that } \\
\text { our hospital pays great attention to patient safety }\end{array}$ & 5=positive & $3.0(1.0)$ & $3.2(1.0)$ & (784) $-3.698^{*}$ & 0.20 \\
\hline $\begin{array}{l}\text { Do you have an individual influence on how well } \\
\text { patient safety is implemented at the workplace }\end{array}$ & $1=$ positive & $3.2(0.9)$ & $2.9(1.0)$ & (910) $4.558^{*}$ & -0.32 \\
\hline Occupational safety climate & $\begin{array}{l}\text { Interpretation } \\
\text { ( } 1=\text { minimum value, } \\
5=\text { maximum value) }\end{array}$ & $\begin{array}{l}\text { Mean (SD) } \\
\text { (nurses=543) }\end{array}$ & $\begin{array}{l}\text { Mean (SD) } \\
\text { (physicians=369) }\end{array}$ & (df) t-value ${ }^{1}$ & $\mathrm{~d}_{\text {Cohen }}$ \\
\hline
\end{tabular}


Table 3 Descriptive statistics, results of the student's t test and effect size comparing answers by nurses and physicians (Continued)

\begin{tabular}{|c|c|c|c|c|c|}
\hline \multicolumn{6}{|l|}{ TWINS Occupational Safety } \\
\hline Supervisor support for occupational safety & $5=$ positive & $3.5(0.8)$ & $3.4(0.8)$ & (910) 1.050 & -0.13 \\
\hline $\begin{array}{l}\text { My direct supervisor openly addresses problems } \\
\text { concerning occupational safety in our hospital }\end{array}$ & 5=positive & $3.2(0.9)$ & $3.2(0.9)$ & (910) 0.869 & 0.00 \\
\hline $\begin{array}{l}\text { My direct supervisor focuses more on occupational } \\
\text { safety than a year ago }\end{array}$ & $5=$ positive & $2.8(0.9)$ & $2.7(0.9)$ & (910) 0.628 & -0.11 \\
\hline $\begin{array}{l}\text { It is important to my direct supervisor that our } \\
\text { hospital pays great attention to occupational safety }\end{array}$ & 5=positive & $3.3(0.9)$ & $3.2(1.0)$ & (910) $2.299^{*}$ & -0.11 \\
\hline $\begin{array}{l}\text { Hospital management openly addresses problems } \\
\text { concerning occupational safety in our hospital }\end{array}$ & $5=$ positive & $2.9(0.9)$ & $3.1(0.9)$ & (910) $-3.337^{*}$ & 0.22 \\
\hline $\begin{array}{l}\text { Hospital management focuses more on occupational } \\
\text { safety than a year ago }\end{array}$ & $5=$ positive & $2.7(0.9)$ & $2.8(0.9)$ & (910) -1.936 & 0.11 \\
\hline $\begin{array}{l}\text { It is important to the Hospital management that our } \\
\text { hospital pays great attention to occupational safety }\end{array}$ & $5=$ positive & $2.9(0.9)$ & $3.1(1.0)$ & $(766)-2.720^{*}$ & 0.21 \\
\hline $\begin{array}{l}\text { Do you have an individual influence on how well } \\
\text { occupational safety is implemented at the workplace }\end{array}$ & $1=$ positive & $3.3(0.9)$ & $3.3(1.0)$ & (910) 0.893 & 0.00 \\
\hline Occupational safety climate & $\begin{array}{l}\text { Interpretation } \\
(1=\text { minimum value, } \\
5=\text { maximum value })\end{array}$ & $\begin{array}{l}\text { Mean (SD) } \\
\text { (nurses=560) }\end{array}$ & $\begin{array}{l}\text { Mean (SD) } \\
\text { (physicians=372) }\end{array}$ & (df) t-value ${ }^{1}$ & $d_{\text {Cohen }}$ \\
\hline \multicolumn{6}{|l|}{ Outcome scales - self constructed indices } \\
\hline $\begin{array}{l}\text { Subjective assessment of specific protective measures } \\
\text { (behaviour \& regulations) related to infectious diseases }\end{array}$ & $1=$ positive & $1.8(0.6)$ & $1.8(0.6)$ & (930) -1.132 & 0.00 \\
\hline $\begin{array}{l}\text { Subjective assessment of occupational safety measures } \\
\text { initiated by the employer, related to own safety }\end{array}$ & $1=$ positive & $1.7(0.6)$ & $2.0(0.6)$ & (930) $-8.328^{*}$ & 0.50 \\
\hline Personal perception of the frequency of occupational risks & $5=$ positive & $3.2(0.8)$ & $3.5(0.7)$ & (853) $-5.608^{*}$ & 0.39 \\
\hline
\end{tabular}

Notes: ${ }^{1} p$-value* $\leq .05$

\section{'Additional file 1': Revised version}

We corrected the values for the scale "Social relations". We also discovered another minor error (concerning the scale influence at work) and corrected the value too. The table with the corrected values is shown below:

\begin{tabular}{|c|c|c|c|c|c|}
\hline $\begin{array}{l}\text { Psychosocial } \\
\text { working } \\
\text { conditions }\end{array}$ & $\begin{array}{l}\text { Interpretation } \\
(0=\text { minimum } \\
\text { value, } 100= \\
\text { maximum } \\
\text { value })\end{array}$ & $\begin{array}{l}\text { Mean } \\
\text { (SD) } \\
\text { (hospital } \\
1=573 \text { ) }\end{array}$ & $\begin{array}{l}\text { Mean } \\
\text { (SD) } \\
\text { (hospital } \\
2=418 \text { ) }\end{array}$ & $\begin{array}{l}\text { (df) } t- \\
\text { value }\end{array}$ & $\mathrm{d}_{\text {Coher }}$ \\
\hline \multicolumn{6}{|c|}{ Copenhagen Psychosocial Questionnaire (COPSOQ) } \\
\hline Quantitative demands & high=negative & $68.4(13.7)$ & $68.9(14,5)$ & $\begin{array}{l}(989) \\
-0.568\end{array}$ & 0.04 \\
\hline Emotional demands & high=negative & $65.1(17.7)$ & $63.1(17,8)$ & $\begin{array}{l}(989) \\
1.742\end{array}$ & -0.11 \\
\hline Work-privacy-conflict & high=negative & $62.2(25.5)$ & $66.0(24,8)$ & $\begin{array}{l}(989) \\
-2.332^{*}\end{array}$ & 0.15 \\
\hline Influence at work & high=positive & $36.1(19.1)$ & $38.9(18,5)$ & $\begin{array}{l}(989) \\
-2.295^{*}\end{array}$ & 0.15 \\
\hline Degree of freedom at work & high=positive & $39.8(18.5)$ & $40.9(18,4)$ & $\begin{array}{l}(989) \\
-0.926\end{array}$ & 0.06 \\
\hline Possibilities for development & high=positive & $75.2(16.2)$ & $74.3(15,5)$ & $\begin{array}{l}(989) \\
0.896\end{array}$ & -0.06 \\
\hline Meaning of work & high=positive & $80.6(16.0)$ & $78.5(17,7)$ & $\begin{array}{l}(989) \\
1.918\end{array}$ & -0.13 \\
\hline Workplace commitment & high=positive & $55.0(18.8)$ & $51.8(21,7)$ & $\begin{array}{l}(820) \\
2.447^{*}\end{array}$ & -0.16 \\
\hline Predictability & high=positive & $54.7(17.0)$ & $50.7(18,5)$ & $\begin{array}{l}(989) \\
3.452^{*}\end{array}$ & -0.23 \\
\hline Role clarity & high=positive & $74.3(15.3)$ & $71.6(15,9)$ & $\begin{array}{l}(989) \\
2.746^{*}\end{array}$ & -0.17 \\
\hline Role conflicts & high=negative & $47.3(17.3)$ & $49.9(18,9)$ & $\begin{array}{l}(989) \\
-2.267^{*}\end{array}$ & 0.15 \\
\hline
\end{tabular}

\begin{tabular}{|c|c|c|c|c|c|}
\hline Feedback & high=positive & $40.1(20.7)$ & $43.5(22,0)$ & $\begin{array}{l}(866) \\
-2.418^{*}\end{array}$ & 0.16 \\
\hline Social support & high=positive & $66,0(16.4)$ & $65.4(17,7)$ & $\begin{array}{l}(858) \\
0.587\end{array}$ & -0.04 \\
\hline Social relations & high=positive & $55.4(20.5)$ & $55.9(20.8)$ & $\begin{array}{l}(989) \\
-0.401\end{array}$ & 0.02 \\
\hline $\begin{array}{l}\text { Sense of } \\
\text { community }\end{array}$ & high=positive & $78.1(14.8)$ & $76.2(15,2)$ & $\begin{array}{l}(989) \\
1.949\end{array}$ & -0.13 \\
\hline \multicolumn{6}{|c|}{ Outcome scale - Copenhagen Psychosocial Questionnaire (COPSOQ) } \\
\hline Job satisfaction & high=positive & $70.4(11.1)$ & $69.3(11.7)$ & $\begin{array}{l}(989) \\
1.475\end{array}$ & -0.10 \\
\hline \multicolumn{6}{|c|}{ Outcome scale - Copenhagen Burnout Inventory (CBI, adapted client-related burnout) } \\
\hline Patient related burnout & high=negative & $33.4(17.4)$ & $32.1(18.0)$ & $\begin{array}{l}(989) \\
1.141\end{array}$ & -0.07 \\
\hline Leadership & $\begin{array}{l}\text { Interpretation } \\
(0 / 1=\text { minimum } \\
\text { value, } 100 / 5= \\
\text { maximum } \\
\text { value })\end{array}$ & $\begin{array}{l}\text { Mean } \\
\text { (SD) } \\
\text { (hospital } \\
1=544 \text { ) }\end{array}$ & $\begin{array}{l}\text { Mean } \\
\text { (SD) } \\
\text { (hospital } \\
2=409 \text { ) }\end{array}$ & $\begin{array}{l}\text { (df) t- } \\
\text { value }^{1}\end{array}$ & $d_{\text {Cohen }}$ \\
\hline \multicolumn{6}{|c|}{ Transformational Leadership Inventory (TLI short) } \\
\hline Transformational leadership & $5=$ positive & $3.2(0.8)$ & $3.2(0.8)$ & $\begin{array}{l}(951) \\
0.191\end{array}$ & 0.00 \\
\hline \multicolumn{6}{|c|}{ Copenhagen Psychosocial Questionnaire (COPSOQ) } \\
\hline Quality of leadership & high=positive & $52.7(22.6)$ & $51.0(23.4)$ & $\begin{array}{l}(951) \\
1.095\end{array}$ & -0.07 \\
\hline Patient safety climate & Interpretation & Mean & Mean & (df) $\mathrm{t}-$ & $d_{C_{C}}$ \\
\hline
\end{tabular}




\section{(Continued)}

\begin{tabular}{|c|c|c|c|c|c|}
\hline & $\begin{array}{l}(1=\text { minimum } \\
\text { value, } \\
5= \\
\text { maximum } \\
\text { value) }\end{array}$ & $\begin{array}{l}\text { (SD) } \\
\text { (hospital } \\
1=560 \text { ) }\end{array}$ & $\begin{array}{l}\text { (SD) } \\
\text { (hospital } \\
2=414 \text { ) }\end{array}$ & value $^{1}$ & \\
\hline \multicolumn{6}{|c|}{ Hospital Survey on Patient Safety Culture (HSPSC-D) } \\
\hline Staffing & $5=$ positive & $2.5(0.8)$ & $2.6(0.8)$ & $\begin{array}{l}(972) \\
-0.965\end{array}$ & 0.13 \\
\hline Organizational learning & $5=$ positive & $3.1(0.7)$ & $3.0(0.7)$ & $\begin{array}{l}(972) \\
0.758\end{array}$ & -0.14 \\
\hline Communication openness & $5=$ positive & $3.6(0.7)$ & $3.5(0.7)$ & $\begin{array}{l}(972) \\
2.207^{*}\end{array}$ & -0.14 \\
\hline $\begin{array}{l}\text { Feedback \& communication } \\
\text { about error }\end{array}$ & $5=$ positive & $3.4(0.8)$ & $3.3(0.9)$ & $\begin{array}{l}(972) \\
2.315^{*}\end{array}$ & -0.12 \\
\hline $\begin{array}{l}\text { Nonpunitive response } \\
\text { to error }\end{array}$ & $5=$ positive & $3.5(0.8)$ & $3.2(0.8)$ & $\begin{array}{l}(843) \\
4.585^{*}\end{array}$ & -0.38 \\
\hline Teamwork within units & $5=$ positive & $3.4(0.6)$ & $3.3(0.6)$ & $\begin{array}{l}(972) \\
1.669\end{array}$ & -0.17 \\
\hline Teamwork across units & $5=$ positive & $3.1(0.6)$ & $3.0(0.6)$ & $\begin{array}{l}(972) \\
1.800\end{array}$ & -0.17 \\
\hline Handoffs \& transitions & $5=$ positive & $3.1(0.6)$ & $3.0(0.6)$ & $\begin{array}{l}(972) \\
2.187^{*}\end{array}$ & -0.17 \\
\hline $\begin{array}{l}\text { Supervisor/ manager } \\
\text { expectations }\end{array}$ & $5=$ positive & $3.3(0.7)$ & $3.3(0.7)$ & $\begin{array}{l}(972) \\
-0.273\end{array}$ & 0.00 \\
\hline $\begin{array}{l}\text { Management support } \\
\text { for patient safety }\end{array}$ & $5=$ positive & $2.8(0.9)$ & $2.7(0.9)$ & $\begin{array}{l}(972) \\
1.579\end{array}$ & -0.11 \\
\hline \multicolumn{6}{|c|}{ Outcome scales - Hospital Survey on Patient Safety Culture (HSPSC-D) } \\
\hline $\begin{array}{l}\text { Frequency of event } \\
\text { reported }\end{array}$ & $5=$ positive & $3.0(1.0)$ & $3.0(1.0)$ & $\begin{array}{l}(972) \\
-0.191\end{array}$ & 0.00 \\
\hline $\begin{array}{l}\text { Overall perceptions of patient } \\
\text { safety }\end{array}$ & $5=$ positive & $3.0(0.8)$ & $3.1(0.8)$ & $\begin{array}{l}(972) \\
-1.262\end{array}$ & 0.13 \\
\hline Patient safety grade & $1=$ positive & $2.8(0.8)$ & $2.8(0.7)$ & $\begin{array}{l}(972) \\
0.405\end{array}$ & 0.00 \\
\hline $\begin{array}{l}\text { Safety grade in the medication } \\
\text { process }\end{array}$ & 1=positive & $2.8(0.7)$ & $3.0(0.8)$ & $\begin{array}{l}(972) \\
-2.730^{*}\end{array}$ & 0.27 \\
\hline Patient safety climate & $\begin{array}{l}\text { Interpretation } \\
(1=\text { minimum value, } 5= \\
\text { maximum } \\
\text { value })\end{array}$ & $\begin{array}{l}\text { Mean } \\
\text { (SD) } \\
\text { (hospital } \\
1=544 \text { ) }\end{array}$ & $\begin{array}{l}\text { Mean } \\
\text { (SD) } \\
\text { (hospital } \\
2=409 \text { ) }\end{array}$ & $\begin{array}{l}\text { (df) } t- \\
\text { value }^{1}\end{array}$ & $d_{\text {Cohen }}$ \\
\hline \multicolumn{6}{|l|}{ TWINS Patient Safety (TWINS-PS) } \\
\hline $\begin{array}{l}\text { Supervisor support for patient } \\
\text { safety }\end{array}$ & $5=$ positive & $3.5(0.8)$ & $3.5(0.8)$ & $\begin{array}{l}(951) \\
0.702\end{array}$ & 0.00 \\
\hline $\begin{array}{l}\text { My direct supervisor openly } \\
\text { addresses problems concerning } \\
\text { patient safety in our hospital }\end{array}$ & $5=$ positive & $3.3(0.9)$ & $3.3(0.9)$ & $\begin{array}{l}(951) \\
-0.794\end{array}$ & 0.00 \\
\hline $\begin{array}{l}\text { My direct supervisor focuses } \\
\text { more on patient safety than a } \\
\text { year ago }\end{array}$ & $5=$ positive & $2.8(0.9)$ & $2.8(1.0)$ & $\begin{array}{l}(847) \\
0.191\end{array}$ & 0.00 \\
\hline $\begin{array}{l}\text { It is important to my direct } \\
\text { supervisor that our hospital pays } \\
\text { great attention to patient safety }\end{array}$ & $5=$ positive & $3.5(0.9)$ & $3.5(0.9)$ & $\begin{array}{l}(951) \\
0.380\end{array}$ & 0.00 \\
\hline $\begin{array}{l}\text { Hospital management openly } \\
\text { addresses problems concerning } \\
\text { patient safety in our hospital }\end{array}$ & $5=$ positive & $2.9(0.8)$ & $2.8(0.9)$ & $\begin{array}{l}(864) \\
2.555^{*}\end{array}$ & -0.12 \\
\hline $\begin{array}{l}\text { Hospital management focuses } \\
\text { more on patient safety than a } \\
\text { year ago }\end{array}$ & $5=$ positive & $2.7(0.9)$ & $2.8(0.9)$ & $\begin{array}{l}(951) \\
-0.382\end{array}$ & 0.11 \\
\hline $\begin{array}{l}\text { It is important to the Hospital } \\
\text { management that our hospital } \\
\text { pays great attention to patient } \\
\text { safety }\end{array}$ & $5=$ positive & $3.2(0.9)$ & $3.0(1.0)$ & $\begin{array}{l}(951) \\
2.344^{*}\end{array}$ & -0.21 \\
\hline $\begin{array}{l}\text { Do you have an individual } \\
\text { influence on how well patient } \\
\text { safety is implemented at the } \\
\text { workplace }\end{array}$ & $1=$ positive & $3.1(0.9)$ & $3.0(1.0)$ & $\begin{array}{l}(951) \\
1.434\end{array}$ & -0.11 \\
\hline Occupational safety climate & $\begin{array}{l}\text { Interpretation } \\
(1=\text { minimum } \\
\text { value, } 5= \\
\text { maximum } \\
\text { value })\end{array}$ & $\begin{array}{l}\text { Mean } \\
\text { (SD) } \\
\text { (hospital } \\
1=544 \text { ) }\end{array}$ & $\begin{array}{l}\text { Mean } \\
\text { (SD) } \\
\text { (hospital } \\
2=409 \text { ) }\end{array}$ & $\begin{array}{l}\text { (df) } t- \\
\text { value }^{1}\end{array}$ & $d_{\text {Cohen }}$ \\
\hline \multicolumn{6}{|c|}{ TWINS Occupational Safety (TWINS-OS) } \\
\hline $\begin{array}{l}\text { Supervisor support for } \\
\text { occupational safety }\end{array}$ & $5=$ positive & $3.5(0.8)$ & $3.4(0.8)$ & $\begin{array}{l}(951) \\
0.736\end{array}$ & -0.13 \\
\hline $\begin{array}{l}\text { My direct supervisor openly } \\
\text { addresses problems concerning } \\
\text { occupational safety in our } \\
\text { hospital }\end{array}$ & $5=$ positive & $3.3(0.9)$ & $3.2(0.9)$ & $\begin{array}{l}(951) \\
1.683\end{array}$ & -0.11 \\
\hline $\begin{array}{l}\text { My direct supervisor focuses } \\
\text { more on occupational safety than }\end{array}$ & $5=$ positive & $2.8(0.9)$ & $2.8(0.9)$ & $\begin{array}{l}(853) \\
0.852\end{array}$ & 0.00 \\
\hline
\end{tabular}

(Continued)

\begin{tabular}{|c|c|c|c|c|c|}
\hline \multicolumn{6}{|l|}{ a year ago } \\
\hline $\begin{array}{l}\text { It is important to my direct } \\
\text { supervisor that our hospital pays } \\
\text { great attention to occupational } \\
\text { safety }\end{array}$ & $5=$ positive & $3.3(0.9)$ & $3.2(1.0)$ & $\begin{array}{l}(951) \\
1.252\end{array}$ & -0.11 \\
\hline $\begin{array}{l}\text { Hospital management openly } \\
\text { addresses problems concerning } \\
\text { occupational safety in our } \\
\text { hospital }\end{array}$ & $5=$ positive & $3.1(0.9)$ & $2.9(0.9)$ & $\begin{array}{l}(951) \\
2.470^{*}\end{array}$ & -0.22 \\
\hline $\begin{array}{l}\text { Hospital management focuses } \\
\text { more on occupational safety than } \\
\text { a year ago }\end{array}$ & $5=$ positive & $2.7(0.9)$ & $2.7(1.0)$ & $\begin{array}{l}(820) \\
0.220\end{array}$ & 0.00 \\
\hline $\begin{array}{l}\text { It is important to the Hospital } \\
\text { management that our hospital } \\
\text { pays great attention to } \\
\text { occupational safety }\end{array}$ & $5=$ positive & $3.1(1.0)$ & $3.0(1.0)$ & $\begin{array}{l}(951) \\
1.193\end{array}$ & -0.10 \\
\hline $\begin{array}{l}\text { Do you have an individual } \\
\text { influence on how well } \\
\text { occupational safety is } \\
\text { implemented at the workplace }\end{array}$ & $1=$ positive & $3.3(0.9)$ & $3.3(1.0)$ & $\begin{array}{l}(951) \\
0.826\end{array}$ & 0.00 \\
\hline Occupational safety climate & $\begin{array}{l}\text { Interpretation } \\
(1=\text { minimum value, } 5= \\
\text { maximum } \\
\text { value })\end{array}$ & $\begin{array}{l}\text { Mean } \\
\text { (SD) } \\
\text { (hospital } \\
1=560 \text { ) }\end{array}$ & $\begin{array}{l}\text { Mean } \\
\text { (SD) } \\
\text { (hospital } \\
2=413 \text { ) }\end{array}$ & $\begin{array}{l}\text { (df) } t- \\
\text { value }^{1}\end{array}$ & $\mathrm{~d}_{\text {Cohen }}$ \\
\hline \multicolumn{6}{|c|}{ Outcome scales - self constructed indices s } \\
\hline $\begin{array}{l}\text { Subjective assessment of specific } \\
\text { protective measures (behaviour \& } \\
\text { regulations) related to infectious } \\
\text { diseases }\end{array}$ & $1=$ positive & $1.8(0.6)$ & $1.8(0.6)$ & $\begin{array}{l}(971) \\
0.396\end{array}$ & 0.00 \\
\hline $\begin{array}{l}\text { Subjective assessment of } \\
\text { occupational safety measures } \\
\text { initiated by the employer, related } \\
\text { to own safety }\end{array}$ & $1=$ positive & $1.8(0.6)$ & $1.9(0.6)$ & $\begin{array}{l}(835) \\
-1.632\end{array}$ & 0.17 \\
\hline $\begin{array}{l}\text { Personal perception of the } \\
\text { frequency of occupational risks }\end{array}$ & $5=$ positive & $3.4(0.7)$ & $3.3(0.8)$ & $\begin{array}{l}(825) \\
1.870\end{array}$ & -0.13 \\
\hline
\end{tabular}

Notes: ${ }^{1} p$-value ${ }^{*} \leq .05$

\section{Author details}

'Institute of Occupational and Social Medicine and Health Services Research, University Hospital of Tübingen, Wilhelmstraße 27, 72074 Tübingen,

Germany. ${ }^{2}$ University of Applied Sciences and Arts Northwestern Switzerland, FHNW School of Applied Psychology, Riggenbachstrasse 16, 4600 Olten, Switzerland. ${ }^{3}$ Institute for Clinical Epidemiology and Applied Biometry, University Hospital of Tübingen, Silcherstraße 5, 72076 Tübingen, Germany. ${ }^{4}$ Berlin Institute of Health (BIH), Clinical Research Unit (CRU),

Anna-Louisa-Karsch-Str. 2, 10178 Berlin, Germany. ${ }^{5}$ Institute of Biometry and Clinical Epidemiology, Charité -Universitätsmedizin Berlin, Charitéplatz 1, 10117 Berlin, Germany. Institute for Patient Safety, University Hospital of Bonn, Sigmund-Freud-Str. 25, 53127 Bonn, Germany. ${ }^{7}$ independent consultant, Berlin, Germany.

Published online: 22 January 2020

\section{Reference}

1. Wagner, et al. Healthcare professionals' perspectives on working conditions, leadership, and safety climate: a cross-sectional study. BMC Health Serv Res. 2019;19:53 https://doi.org/10.1186/s12913-018-3862-7. 